Cite this: J. Mater. Chem. C, 2013, 1, 7560

Received 13th August 2013 Accepted 20th September 2013

DOI: $10.1039 / c 3 t c 31593 b$

www.rsc.org/MaterialsC

\section{Transformation from a rod-like to a hockey-stick-like and bent-shaped molecule in 3,4'-disubstituted azobenzene-based mesogens $\uparrow$}

\author{
Martin Horčic, ${ }^{a}$ Václav Kozmík, ${ }^{a}$ Jiři Svoboda, ${ }^{\text {aa }}$ Vladimíra Novotnáb \\ and Damian Pociechac
}

New 3,4'-disubstituted azobenzene-based mesogens with an azo group joined directly to the central benzene core have been synthesised and their liquid crystalline properties studied. Both molecular arms are gradually elongated by the insertion of a number of benzoate units between the central core and a terminal dodecyloxy chain. Then the lengths of the molecular arms and the overall shape are varied from a rod-like to a hockey-stick-like and bent-shape. Typical bent-shaped mesophases and electrooptical switching can be observed upon reaching a sufficient length of the molecular arms. We demonstrated how the mesoscale organization strictly depends on the molecular shape.

\section{Introduction}

Liquid crystalline (LC) materials combine order and mobility on the molecular level and show unique electro-optic properties due to their birefringence and dielectric anisotropy. In the past few years, great effort has been devoted to the recognition of their structure-property relationship. Bent-shaped mesogens represent a new subclass of a large family of liquid crystals. ${ }^{1-5} \mathrm{~A}$ large variety of bent-shaped mesophases have been recognized and described as $\mathrm{B}_{n}$ phases. In contrast to rod-like systems, for which ferro- and/or antiferroelectricity requires molecular chirality, the close packing of bent-shaped molecules in the smectic layers results in polar properties of the phase and in the structural layer chirality, even though the molecules are nonchiral. Switchable structures with preferable antiferroelectric (AF) ordering can be formed. Among bent-shaped mesophases the most frequently studied is the $\mathrm{B}_{2}$ phase with the tilted bentshaped molecules organized in layers, giving rise to the in-layer polar axis and, therefore, to ferro- or antiferroelectric properties. Bent-shaped materials have also been found to show nematic and columnar phases. The nematic phase created by bent-shaped mesogens attracts attention due to the potential biaxiality. ${ }^{6,7}$

${ }^{a}$ Department of Organic Chemistry, Institute of Chemical Technology, CZ-166 28 Prague 6, Czech Republic. E-mail: Jiri.Svoboda@vscht.cz

${ }^{b}$ Institute of Physics, Academy of Science of the Czech Republic, Na Slovance 2, CZ-182 21 Prague 8, Czech Republic. E-mail: novotna@fzu.cz

${ }^{c}$ Laboratory of Dielectrics and Magnetics, Chemistry Department, Warsaw University, Al. Zwirki $i$ Wigury 101, 02-089 Warsaw, Poland

$\dagger$ Electronic supplementary information (ESI) available. See DOI: $10.1039 / \mathrm{c} 3 \mathrm{tc} 31593 \mathrm{~b}$
It has been documented that the mesomorphic properties of bent-shaped mesogens can be tuned by the type of the central aromatic core, the overall number of aromatic units in the molecular structure, the type and orientation of the linkage groups (ester, imino, azo), the lateral substitution and the length of the terminal alkyl chains..$^{5,8-14}$ Very recently it has been shown ${ }^{15,16}$ that an ester linking group can be successfully replaced by a more rigid amide unit. Furthermore, the azo linking group has also been utilized ${ }^{\mathbf{1 3 , 1 4 , 1 7 - 2 0}}$ for the design of the bent-shaped compounds. In most cases the azo group was placed in the outer position of the lengthened arm(s). Recently, Nagaveni et al. ${ }^{21}$ have presented azo-functionalized bent-shaped compounds and found that the presence of the $-\mathrm{N}=\mathrm{N}$ - linkage at different locations in the molecular architecture does not seem to have much effect on the mesomorphic behaviour. On the other hand, the molecular shape and/or length of the ending chain are essential in the design of bent-shaped mesogens.

Recently, many non-symmetrically bent mesogens have also been investigated. In contrast to the bent-shaped molecules with two arms roughly equal, in hockey-stick-like mesogens the two arms are substantially different in the length. In the first type of hockey-stick-like molecules the bend is introduced by an alkyl or alkoxy chain attached in the meta position of the terminal ring. For such a molecule, tilted smectic phases have been found ${ }^{22,23}$ with both possibilities of tilt orientation in adjacent layers: synclinic $\left(\mathrm{SmC}_{\mathrm{S}}\right)$ and anticlinic $\left(\mathrm{SmC}_{\mathrm{A}}\right)$. In the second group of hockey-stick-like mesogens the molecules are formed by a bent-shaped central core with arms containing different numbers of aromatic rings. The lack of symmetry in the molecular structure can be also introduced by different lengths and/or types of terminal chain. Depending on the 
structural character, hockey-stick-like mesogens can exhibit standard calamitic (rod-like) mesophases, such as nematic (N), smectic SmA and SmC phases and/or mesophases typical for bent-shaped mesogens. Being on the border-line between rodlike and bent-shaped mesogens, hockey-stick-like mesogens represent the most developing field in liquid crystal research and are a subject of considerable interest. ${ }^{21-27}$

Herein, we present a synthesis and mesomorphic study of a series of materials with an azo group joined directly to the central benzene, creating a 3,4'-dihydroxyazobenzene core. We have chosen this type of molecular core as being rigid enough to avoid conformational changes at the central part. We have varied the length of both molecular arms by inserting an additional number of benzene rings. We have prepared a series of materials, the design of which enabled us to change the overall molecular shape from rod-like to bent-core. The types of mesophases and their physical properties were monitored by optical microscopy studies, X-ray diffraction analysis, DSC and electro-optical measurements.

\section{Synthesis}

The synthesis of the target compounds $\mathbf{A} / \boldsymbol{m} / \boldsymbol{n}$ is depicted in Scheme 1. It is based on acylation of the phenols $\mathbf{1}$ or 2, with acid chlorides, which were prepared in situ by the reaction with oxalyl chloride with acids 3, 4 or 5 under standard conditions. The length of the terminal alkyl chains was adjusted to dodecyl $\left(\mathrm{C}_{12} \mathrm{H}_{25}\right)$. While the central core 1 served for the synthesis of compounds $\mathbf{A} / \mathbf{m} / \boldsymbol{n}$, both with identical lengths of the arms $(m=n)$ and non-symmetrical materials $(m \neq n)$, the central core 2 was utilized for preparation of materials $\mathbf{A} / \mathbf{0} / \boldsymbol{n}$. The number $m$ (the left arm) varies from 0 to 3 , the number $n$ (the right arm) can be 1,2 or 3 . The overall number of benzene units grows from $3(\mathbf{A} / \mathbf{0} / \mathbf{1})$ to $8(\mathbf{A} / \mathbf{3} / \mathbf{3})$.
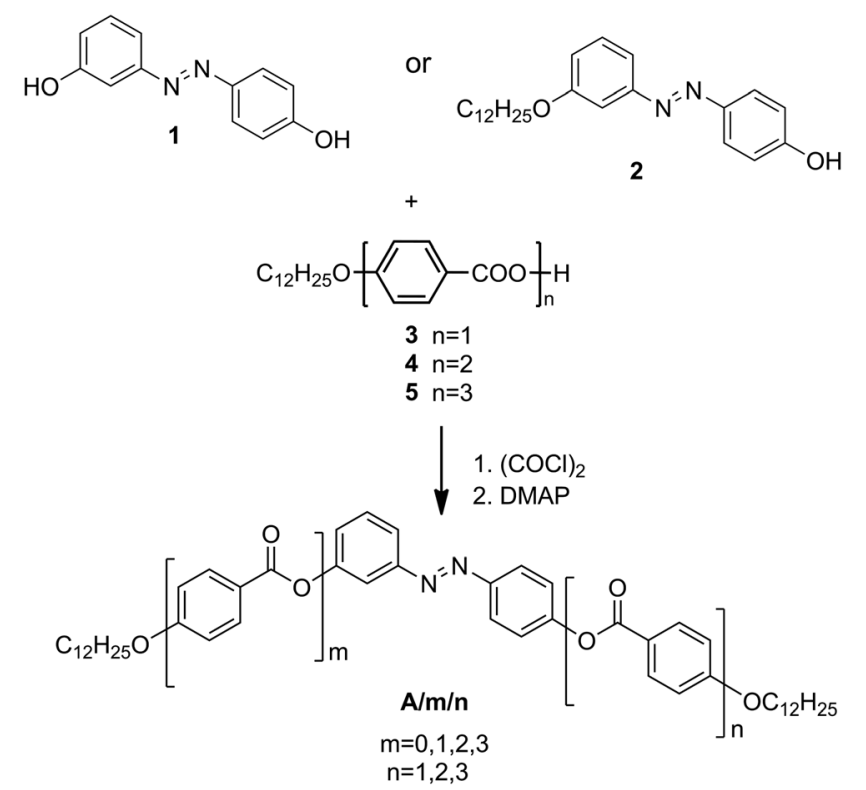

Scheme 1 Synthesis route of materials $A / m / n$.
The synthesis of compounds $\mathbf{1}$, and $\mathbf{2}$, and target compounds $\mathbf{A} / \mathbf{m} / \boldsymbol{n}$, including their detailed molecular structure, all intermediates, and their physico-chemical characterization, is summarized and available in the ESI. $\uparrow$ The synthesis of acids 3 , 4 and 5 was reported elsewhere. ${ }^{\mathbf{8 , 9}}$

\section{Measurements}

Phase transition temperatures were determined by differential scanning calorimetry (DSC) using a Perkin-Elmer 7 (Pyris Diamond) calorimeter. The samples of about 2-5 $\mathrm{mg}$ were hermetically sealed in aluminium pans and placed into the calorimeter chamber, which was filled with nitrogen during the measurement. Temperature and enthalpy values were calibrated using the extrapolated onset temperatures and enthalpy changes for water, indium and zinc. Calorimetric measurements were performed on cooling and heating runs at a rate of $5 \mathrm{Kmin}^{-1}$.

The sequence of phases and phase transition temperatures were determined from the optical textures and their changes, observed under a polarizing optical microscope (POM) Nikon Eclipse E600Pol. The planar cells for texture observation were prepared from glasses with ITO transparent electrodes $(5 \times$ $5 \mathrm{~mm}^{2}$ ), without any surface treatment. The thickness was defined by Mylar sheets as $12 \mu \mathrm{m}$. A commercial cell (AWAT Comp., Warsaw) with a surfactant and thickness of $6 \mu \mathrm{m}$ was applied for the electro-optical studies. The cells were filled in the isotropic phase with studied materials by capillary action. The Linkam LTS E350 heating/cooling stage with a TMS 93 temperature regulator was used for the temperature control and stabilization within $\pm 0.1 \mathrm{~K}$.

Switching studies were performed using a driving voltage from a Phillips generator PM 5191, accompanied by a linear amplifier, providing a maximum amplitude of $120 \mathrm{~V}$. The switching current $v s$. time profile was recorded with a Tektronix memory oscilloscope DPO4034.

X-ray diffraction (XRD) studies were performed using a Bruker Nanostar system (CuK $\alpha$ radiation, Vantec 2000 area detector, MRI TCPU $\mathrm{H}$ heating stage) working in transmission mode and a Bruker D8 Discover system (CuK $\alpha$ radiation, scintillation counter, DCS350 heating stage) working in reflection mode. In both systems the temperature stability was $0.1 \mathrm{~K}$. Powder samples (for Nanostar) were prepared in thin-walled glass capillaries $(1.5 \mathrm{~mm}$ diameter $)$, aligned samples for experiments in reflection mode were prepared as thin films on a heated silicon wafer.

\section{Results}

DSC studies were performed for all prepared compounds. The phase transition temperatures and associated enthalpy changes are summarized in Table 1 . The DSC thermographs are shown in Fig. 1a-c for representative materials $\mathbf{A} / \mathbf{0} / \mathbf{2}, \mathbf{A} / \mathbf{1} / \mathbf{3}$ and $\mathbf{A} / \mathbf{2} / \mathbf{3}$, respectively. Phases and phase sequences have been identified by texture observation under a polarizing optical microscope and confirmed by X-ray measurements (see below). With the exception of non-mesogenic $\mathbf{A} / \mathbf{0} / \mathbf{1}, \mathbf{A} / \mathbf{1} / \mathbf{1}$ and $\mathbf{A} / \mathbf{1} / \mathbf{2}$, all other 


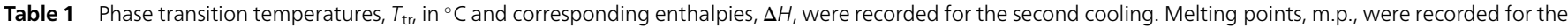

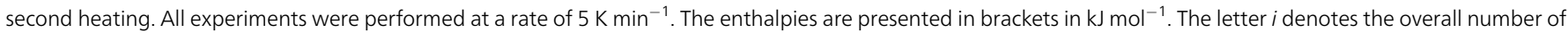
benzene rings in the molecule

\begin{tabular}{|c|c|c|c|c|c|c|c|c|c|c|}
\hline $\mathrm{A} / m / n$ & $i$ & M.p. $(\Delta H)$ & $T_{\operatorname{tr}}(\Delta H)$ & $\mathrm{M}_{3}$ & $T_{\operatorname{tr}}(\Delta H)$ & $\mathbf{M}_{2}$ & $T_{\operatorname{tr}}(\Delta H)$ & $\mathrm{M}_{1}$ & $T_{\mathrm{tr}}(\Delta H)$ & Iso \\
\hline $\mathrm{A} / \mathbf{0} / \mathbf{1}$ & 3 & $62(+71.0)$ & $51(-65.7)$ & & & & & - & & $\bullet$ \\
\hline $\mathrm{A} / \mathbf{0} / 2$ & 4 & $86(+94.5)$ & $63(-68.5)$ & $\mathrm{SmC}_{\mathrm{A}}$ & $108(-0.02)$ & $\mathrm{SmC}$ & $139(-0.35)$ & $\mathrm{SmA}$ & $145(-6.0)$ & $\bullet$ \\
\hline $\mathbf{A} / \mathbf{0} / 3$ & 5 & $104(+34.4)$ & $99(-29.7)$ & & & $\mathrm{SmC}$ & $223(-0.25)$ & $\mathrm{SmA}$ & $234(-3.04)$ & $\bullet$ \\
\hline $\mathbf{A} / \mathbf{1} / \mathbf{1}$ & 4 & $84(+49.4)$ & $66(-47.0)$ & & & & & - & & $\bullet$ \\
\hline $\mathbf{A} / \mathbf{1} / \mathbf{2}$ & 5 & $121(+63.8)$ & $116(-42.9)$ & & & & & - & & $\bullet$ \\
\hline $\mathbf{A} / \mathbf{1} / \mathbf{3}$ & 6 & $131(+40.9)$ & $123(-39.5)$ & $\mathrm{SmC}_{\mathrm{A}}$ & $158(-0.08)$ & $\mathrm{SmC}$ & $189(-0.74)$ & $\mathrm{N}$ & $191(-1.35)$ & $\bullet$ \\
\hline $\mathbf{A} / 2 / 1$ & 5 & $115(+50.2)$ & $91(-30.1)$ & & & & & $\mathrm{SmC}_{\mathrm{A}} \mathrm{P}_{\mathrm{A}}$ & $112(-20.1)$ & $\bullet$ \\
\hline $\mathrm{A} / 2 / 2$ & 6 & $111(+49.3)$ & $69(-15.4)$ & & & $\mathrm{SmC}_{\mathrm{A}} \mathrm{P}_{\mathrm{A}}$ & $138(-0.69)$ & $\mathrm{B}_{1}$ & $156(-19.1)$ & $\bullet$ \\
\hline $\mathbf{A} / 2 / 3$ & 7 & $132(+32.8)$ & $113(-29.7)$ & & & & & $\mathrm{B}_{1}$ & $193(-18.5)$ & $\bullet$ \\
\hline $\mathbf{A} / \mathbf{3} / \mathbf{1}$ & 6 & $118(+27.9)$ & $102(-25.8)$ & & & & & $\mathrm{B}_{1}$ & $154(-19.1)$ & $\bullet$ \\
\hline $\mathbf{A} / 3 / 2$ & 7 & $131(+50.2)$ & $113(-29.5)$ & & & & & $\mathrm{B}_{1}$ & $217(-21.3)$ & $\bullet$ \\
\hline $\mathbf{A} / 3 / 3$ & 8 & $151(+31.6)$ & $136(-31.4)$ & & & & & $\mathrm{B}_{1}$ & $245(-12.3)$ & $\bullet$ \\
\hline
\end{tabular}

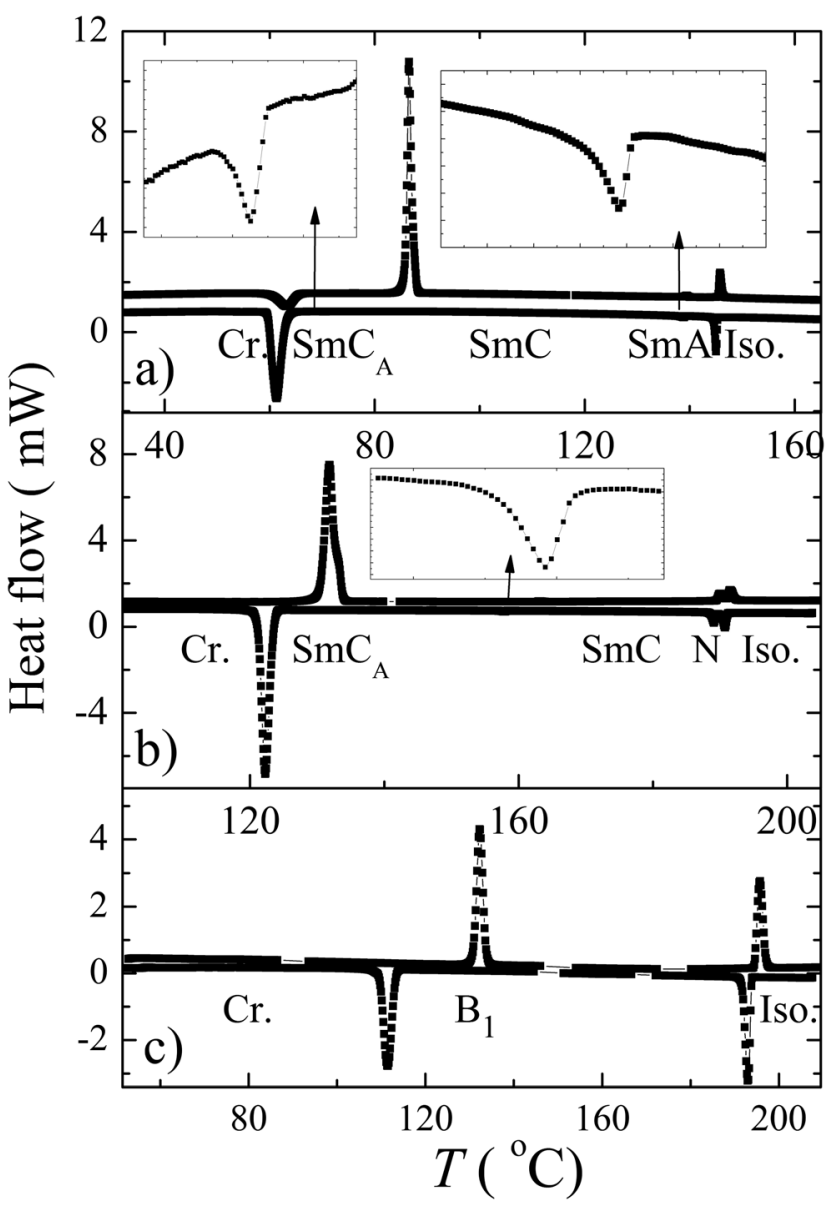

Fig. 1 Temperature dependencies of the heat flow measured by DSC for selected compounds (a) A/0/2, (b) A/1/3 and (c) A/2/3. The phase transitions which are not properly recognizable are marked by arrows and presented in enlarged views in the insets.

compounds exhibited the formation of at least one mesophase. All observed mesophases have enantiotropic character except for $\mathbf{A} / \mathbf{0} / \mathbf{1}$ (where the SmCP phase is monotropic, i.e. is observed only on cooling). As follows from Table 1, the studied materials can be divided into two groups. Compounds with $m=0$ or 1 , in which the molecules can be considered as rod-like or hockeystick-like type, form calamitic mesophases. For all compounds with $m \geq 2$ bent-shaped mesophases are observed.

Mesogens $\mathbf{A} / \mathbf{0} / \mathbf{2}, \mathbf{A} / \mathbf{0} / \mathbf{3}$ and $\mathbf{A} / \mathbf{1} / \mathbf{3}$ exhibit the nematic (N), smectic A (SmA), synclinic smectic $\mathrm{C}(\mathrm{SmC})$ and anticlinic smectic $\mathrm{C}\left(\mathrm{SmC}_{\mathrm{A}}\right)$ phases (Table 1). Marble-like and fan-shaped textures are observed in the nematic and smectic phases, respectively. For $\mathbf{A} / \mathbf{1} / \mathbf{3}$ a planar texture in the nematic, $\mathrm{SmC}$ and $\mathrm{SmC}_{\mathrm{A}}$ phase, revealed in a commercial cell $(6 \mu \mathrm{m})$ upon cooling from the isotropic phase, is shown in Fig. 2a-c, respectively. Surprisingly, rather complicated fan-shaped textures for the tilted SmC phase were observed when we increased the thickness of the cell. We can identify a twist in the broken fan-shaped texture (see Fig. 3 for A/0/3). Domains with twisted structures have been found, which provides evidence for the separation of molecular conformers differing in axial chirality. The cell thickness is very important, for thin enough samples the uniform organization of the molecules through the cell profile is more advantageous, whereas for thicker samples the twisted structure is preferred because the twist elastic energy dominates over the surface energy.

$\mathrm{X}$-ray measurements confirmed the mesophase identification of $\mathbf{A} / \mathbf{0} / \mathbf{2}, \mathbf{A} / \mathbf{0} / \mathbf{3}$ and $\mathbf{A} / \mathbf{1} / \mathbf{3}$. In the nematic phase of the $\mathbf{A} / \mathbf{1} / \mathbf{3}$ compound a weak diffused signal in a small angle range was recorded, which can be attributed to a mean intermolecular distance along the long molecular axis (molecular length). In the smectic phases a sharp peak was detected in a small-angle diffraction range and a diffuse scattering maximum in the wide angle region. This provides evidence for a lamellar structure with liquid-like molecular packing (without in-plane order) within the layers. From the small angle reflections the layer spacing, $d$, was evaluated for all calamitic mesogens A/0/2, A/0/3 and $\mathbf{A} / \mathbf{1} / \mathbf{3}$ (Fig. 4). A slight increase of $d$ was found in the SmA phase upon cooling. Such behaviour is often observed in the SmA phase and can be explained by an increase of the long molecular axis ordering and/or by stretching of the aliphatic chains. An abrupt decrease in $d(T)$ at the $\mathrm{N}-\mathrm{SmC}$ and SmA-SmC phase transitions reflects the tilt of the molecules. The SmC$\mathrm{SmC}_{\mathrm{A}}$ phase transition is accompanied by a slight jump of $d(T)$ 

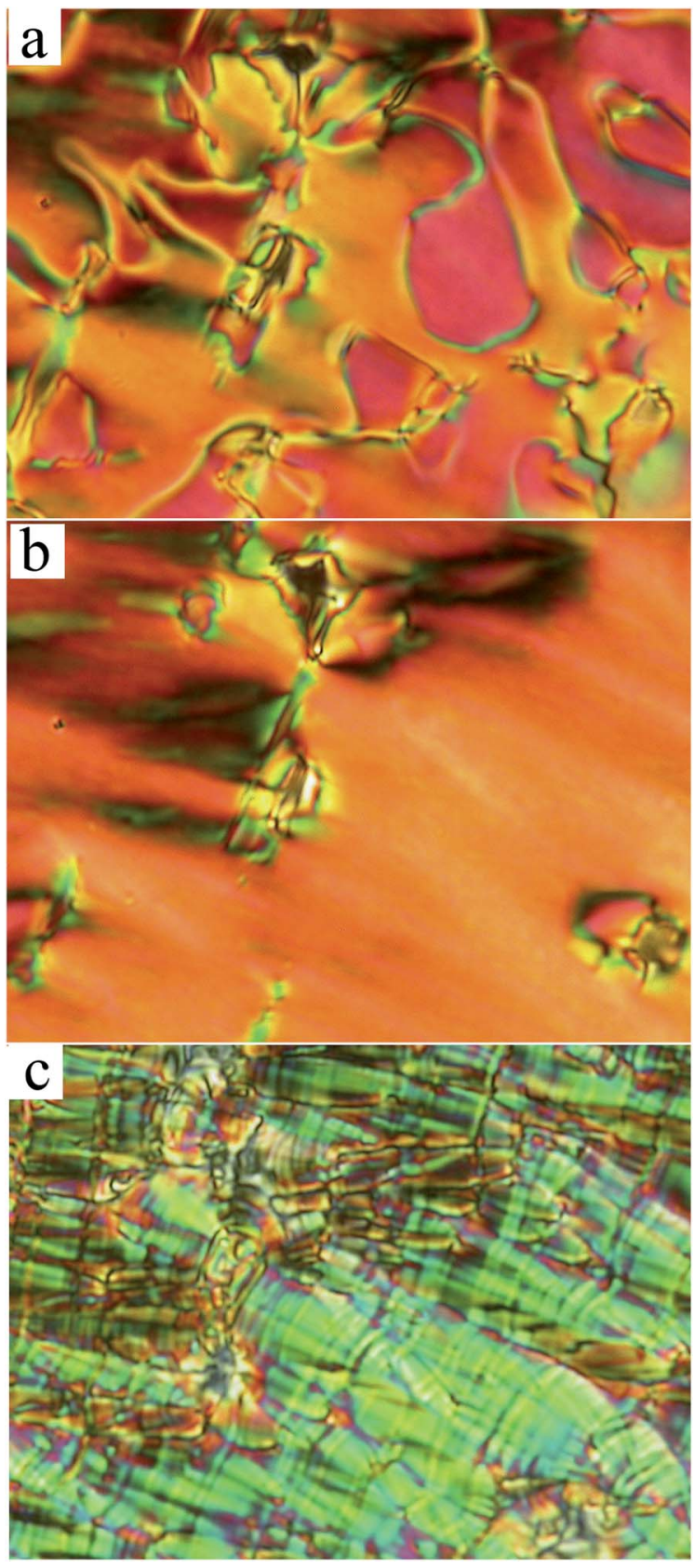

Fig. 2 Planar textures for compound $\mathbf{A} / \mathbf{1 / 3}$ in the (a) nematic, (b) SmC and (c) anticlinic $\mathrm{SmC}_{\mathrm{A}}$ phase. Observations were made using a commercial planar cell of $6 \mu \mathrm{m}$.

and an increase of the X-ray peak intensity. In spite of the continuous tilting of molecules, $d$ values saturate and then start to grow on further cooling. It is probable that the stretching of the aliphatic chains becomes predominant. When comparing the studied mesogens, one can see that $d$ values are proportional to the molecular length (Fig. 4).

Starting from the compound $\mathbf{A} / \mathbf{2} / \mathbf{1}$, the mesophases typical for bent-core molecules appear (Table 1) when lengthening molecular arms. A switchable lamellar SmCP phase has been observed for compound $\mathbf{A} / \mathbf{2} / \mathbf{1}$. A complicated texture with tiny features appeared upon cooling from the isotropic phase. After

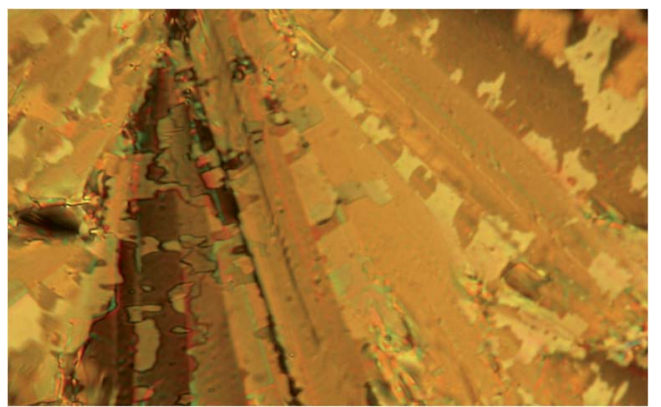

Fig. 3 Photo of the planar texture for $\mathbf{A} / \mathbf{0 / 3}$ in the $\mathrm{SmC}$ phase at $T=150{ }^{\circ} \mathrm{C}$ (the cell thickness is $12 \mu \mathrm{m}$ ).

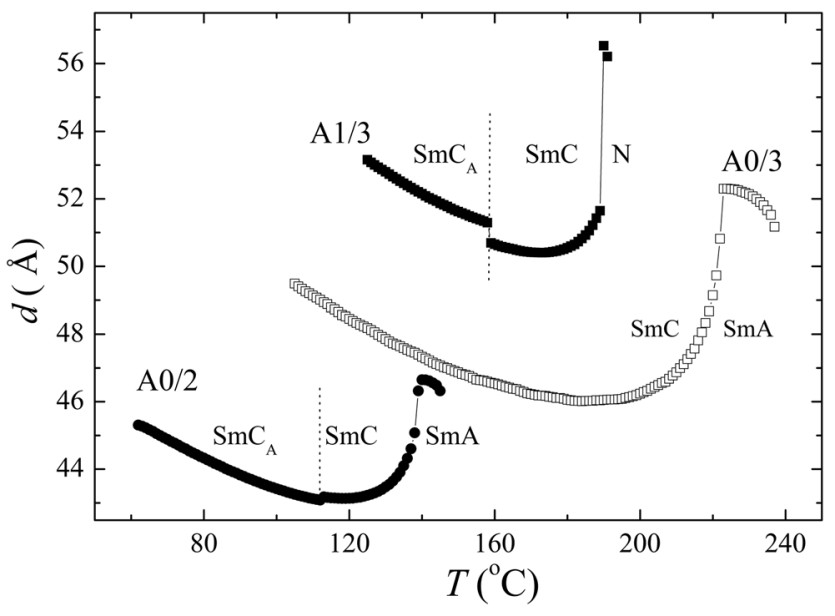

Fig. 4 Temperature dependencies of the layer spacing, $d$, recorded upon cooling for denoted compounds. Phases are indicated.

the application of an electric field with an intensity of about $20 \mathrm{~V} \mu \mathrm{m}^{-1}$ a fan-shaped texture with a low birefringence could be observed under crossed polarizers. Under the electric field the birefringence of the former fan-shaped pattern significantly increases. Additionally, under the applied electric field the extinction position rotates from the orientation parallel to the polarizers by an angle of about $45^{\circ}$. The change of the extinction position is characteristic of the transformation from an anticlinic to synclinic tilt structure under the electric field. Switching properties and dielectric measurements confirm the antiferroelectric character. Two distinct peaks per half-period of the applied electric field within the polarization current profile were measured (see Fig. 5), which is typical for the antiferroelectric phase. Therefore the mesophase can be attributed to the $\mathrm{SmC}_{\mathrm{A}} \mathrm{P}_{\mathrm{A}}$ phase. The spontaneous polarization value $P_{\mathrm{s}}=380 \mathrm{nC}$ $\mathrm{cm}^{-2}$ detected at $T=110{ }^{\circ} \mathrm{C}$ slightly grows within the temperature interval of the $\mathrm{SmC}_{\mathrm{A}} \mathrm{P}_{\mathrm{A}}$ phase. Unfortunately, the studied mesophase has a monotropic character and the crystallization starts when the electric field is applied. From the small angle $\mathrm{X}$-ray measurements the layer spacing, $d$, was evaluated, which was found $(d \approx 35.8 \AA$ ) to be almost temperature independent within the whole temperature range of the $\operatorname{SmC}_{A} \mathrm{P}_{\mathrm{A}}$ phase. When comparing the approximate length of the molecule and 


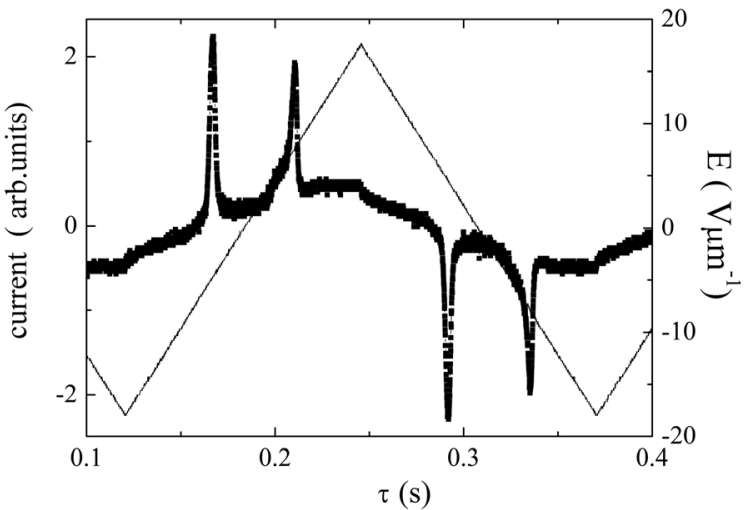

Fig. 5 Switching current profile, detected in the $B_{2}$ phase $\left(T=110^{\circ} \mathrm{C}\right)$ of $\mathbf{A} / \mathbf{2} / \mathbf{1}$

the $d$ value, it is evident that molecules in layers are tilted. Comparing the POM observation, switching properties and $\mathrm{X}$-ray data we can conclude that the observed lamellar phase can be identified only with the $\mathrm{SmC}_{\mathrm{A}} \mathrm{P}_{\mathrm{A}}$ phase.

Compounds $\mathbf{A} / \mathbf{2} / \mathbf{3}, \mathbf{A} / \mathbf{3} / \mathbf{1}, \mathbf{A} / \mathbf{3} / \mathbf{2}$ and $\mathbf{A} / \mathbf{3} / \mathbf{3}$ form a columnar mesophase of $B_{1}$ type, which is built of smectic layer fragments (blocks). The X-ray patterns of the $\mathrm{B}_{1}$ phase were analysed assuming a centred rectangular crystallographic unit cell. Two signals, recorded in a small angle range, were indexed as (11) and (02). The obtained unit cell parameters are summarized in

Table 2 Cell parameters in the columnar $B_{1}$ phase

\begin{tabular}{llll}
\hline & $T /{ }^{\circ} \mathrm{C}$ & $a / \AA$ & $b / \AA$ \\
\hline $\mathbf{A} / \mathbf{2} / 2$ & 150 & 41.0 & 52.2 \\
& 145 & 41.0 & 52.3 \\
$\mathbf{A} / \mathbf{2} / \mathbf{3}$ & 142 & 40.9 & 52.3 \\
& 180 & 41.4 & 57.8 \\
$\mathbf{A} / \mathbf{3} / \mathbf{1}$ & 130 & 40.2 & 58.7 \\
& 140 & 41.7 & 52.9 \\
$\mathbf{A} / 3 / 2$ & 120 & 41.0 & 53.4 \\
& 215 & 43.1 & 57.0 \\
$\mathbf{A} / 3 / 3$ & 140 & 41.4 & 58.4 \\
& 230 & 44.8 & 61.8 \\
& 200 & 44.5 & 62.6 \\
& 150 & 43.3 & 63.6
\end{tabular}

Table 2. For all compounds the $a$ parameter, which reflects the block cross-section length, slightly decreases upon cooling, while the $b$ parameter, which can be attributed to the molecular length, slightly increases. As for the calamitic molecules, this can be explained by a stretching of the aliphatic chains.

An uncommon phase sequence (with a lamellar below a columnar phase upon cooling) was found for compound $\mathbf{A} / \mathbf{2} / \mathbf{2}$. The higher temperature LC mesophase, appearing upon cooling from the isotropic phase, was revealed to be a columnar $B_{1}$ mesophase. Textures, observed under the polarizing microscope, are typical for such a phase, with colourful domain-like and mosaic character (Fig. 6). An applied electric field does not affect the texture, which is a typical feature of a $B_{1}$ phase. In the $B_{1}$ phase, XRD measurements confirmed a $2 \mathrm{D}$ modulated structure (see Table 2). Upon further cooling, at a temperature $T$ $=138{ }^{\circ} \mathrm{C}$, a phase transition was detected by DSC measurements, however, the mosaic-like texture of the $B_{1}$ phase changed only slightly. Nevertheless, under the applied electric field the planar texture changed to the texture typical for a SmCP phase (Fig. 6) below $T=138{ }^{\circ} \mathrm{C}$. Comparing the texture under the field (Fig. 6c) and the texture when the field is switched off (Fig. 6d), one can see that the extinction brushes (regions, where the optical axis is either parallel or perpendicular to the polarization of the incident light when observed in crossed polarizers) rotate by an angle approaching $45^{\circ}$. Such induced changes are characteristic for the transition from the $\mathrm{SmC}_{\mathrm{A}} \mathrm{P}_{\mathrm{A}}$ (without an applied electric field) to the $\mathrm{SmC}_{\mathrm{S}} \mathrm{P}_{\mathrm{F}}$ phase (under an electric field), similar to what we observed for $\mathbf{A} / \mathbf{2} / \mathbf{1}$. When the field is switched off, the low-birefringent texture of the $\mathrm{SmC}_{\mathrm{A}} \mathrm{P}_{\mathrm{A}}$ phase is present and persists until the crystallization upon cooling or up to the phase transition to the $\mathrm{B}_{1}$ phase, which takes place at about $T=139{ }^{\circ} \mathrm{C}$ upon the subsequent heating (Fig. 7). When integrating the polarization current profile (having two peaks per half-period) we obtained the spontaneous polarization value $P_{\mathrm{s}}=300 \mathrm{nC} \mathrm{cm}{ }^{-2}$. Structural parameters of the studied mesophase were established from the X-ray diffraction data (Fig. 8). Below the phase transition at $T=$ $138{ }^{\circ} \mathrm{C}$ only commensurate peaks were detected in the small angle region, confirming a lamellar structure of the studied lowtemperature mesophase. The layer spacing, $d=38.5 \AA$, is almost temperature independent. By comparison with the

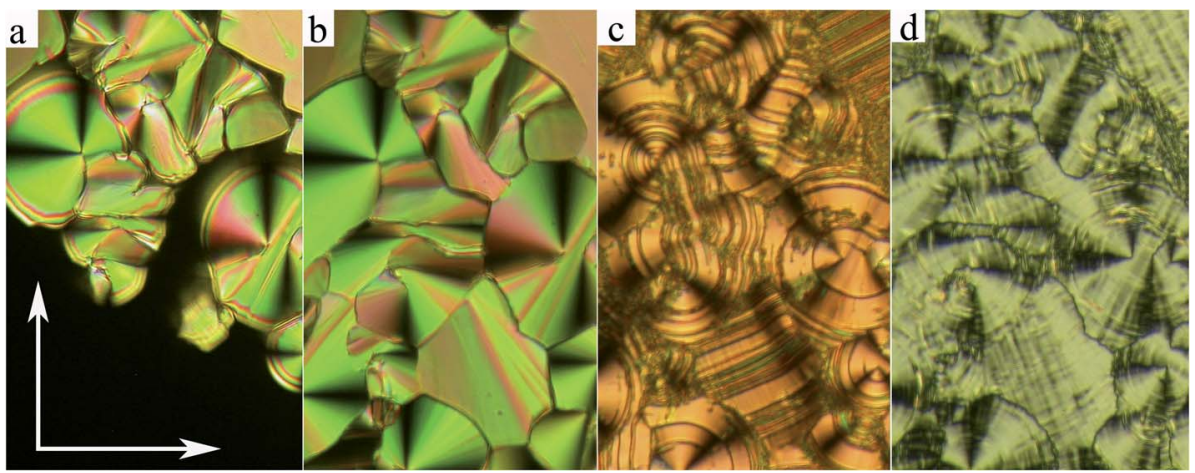

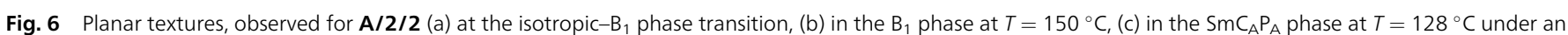
electric field of about $15 \mathrm{~V} \mu \mathrm{m}^{-1}$ and (d) when the field is switched off. Arrows show the orientation of the polarizer and the analyzer. 


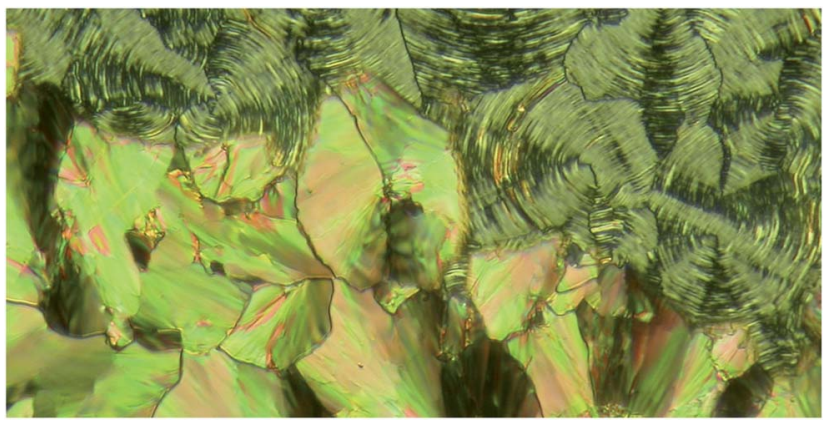

Fig. 7 Planar texture of $\mathbf{A} / \mathbf{2} / \mathbf{2}$ at the $B_{2}-B_{1}$ phase transition, observed upon heating from the $B_{2}$ phase. In the $B_{2}$ phase the sample was subjected to an electric field for several minutes and then the field was switched off. The upper right corner is still in the $\mathrm{SmC}_{A} \mathrm{P}_{A}$ phase and the rest is transformed into the $\mathrm{B}_{1}$ phase.

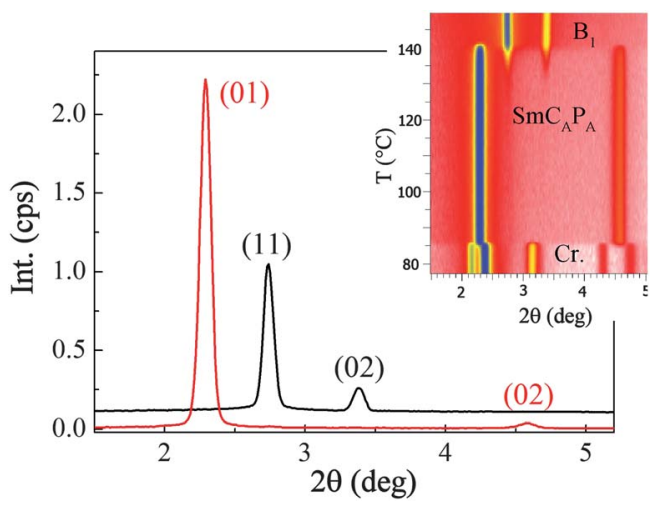

Fig. 8 For $\mathbf{A} / \mathbf{2} / \mathbf{2}$ the $X$-ray intensity, Int., versus the corresponding scattering angle, recorded at the temperature $T=145^{\circ} \mathrm{C}$ (black line) in the $\mathrm{B}_{1}$ phase and $T=$ $130{ }^{\circ} \mathrm{C}$ (red line) in the $\mathrm{SmC}_{A} \mathrm{P}_{\mathrm{A}}$ phase. In the inset (upper right corner) the temperature evolution of the $\mathrm{X}$-ray intensity is presented, starting from the isotropic phase. The colour scale corresponds to the X-ray intensity (blue is for the most intense signal).

estimated length of the molecule ( $52.3 \AA$ A ), the tilt angle can be evaluated as $43^{\circ}$, which agrees well with the angle of the extinction brushes rotation under the applied voltage (Fig. 6). Comparing the X-ray data and the POM observations, we can conclude that the low-temperature phase corresponds to the $\mathrm{SmC}_{\mathrm{A}} \mathrm{P}_{\mathrm{A}}$ phase.

\section{Discussion and conclusions}

We have synthesized 3,4'-disubstituted azobenzene-based liquid crystalline compounds, denoted as $\mathbf{A} / \mathbf{m} / \boldsymbol{n}$. The aim is to compare the mesomorphic properties with respect to the length of the constituent arms, lengthening the central molecular core. Compounds $\mathbf{A} / \mathbf{0} / \mathbf{1}, \mathbf{A} / \mathbf{1} / \mathbf{1}$ and $\mathbf{A} / \mathbf{1} / \mathbf{2}$ do not form any mesophase. It is probable that the self-assembling process is not possible for the studied molecules with a short arm in combination with an asymmetrical bent molecular core. Compounds A/0/2, A/0/3 and $\mathbf{A} / \mathbf{1} / \mathbf{3}$ with only partially asymmetrical bent shapes exhibit calamitic mesophases with no tendency for switching and/or other electro-optical effects under the applied electric field. Nevertheless, the domains with the twisted structure were found in a thick planar cell (Fig. 3), which provides evidence for the separation of conformers based on the axial chirality. The separation of conformers arises spontaneously, but polar surface interactions might enhance this process. The coexistence of various types of uniform and twisted domains is a result of the agreement of their energies. For non-chiral rod-like compounds with an ester linkage group such a phenomenon has been found and described theoretically. ${ }^{27}$ The lack of electro-optical response under the applied electric field in the studied compounds can be explained by the weakness of such a polar effect. We can conclude that a deviation from the molecular linearity and/or symmetry can support a chiral separation.

For mesogens with a lengthened left arm $(m \geq 2)$, bentshaped mesophases were observed, namely the non-switchable columnar $\mathrm{B}_{1}$ phase (for $\mathbf{A} / \mathbf{2} / \mathbf{3}, \mathbf{A} / \mathbf{3} \mathbf{1}, \mathbf{A} / \mathbf{3} / \mathbf{2}$ and $\mathbf{A} / \mathbf{3} / \mathbf{3}$ ) and the polar $\mathrm{SmC}_{\mathrm{A}} \mathrm{P}_{\mathrm{A}}$ phase for $\mathbf{A} / \mathbf{2} / \mathbf{1}$. Surprisingly, for the bent-core mesogen $\mathbf{A} / \mathbf{2} / \mathbf{2}$ we detected an unusual phase sequence: columnar $\mathrm{B}_{1}$-lamellar $\mathrm{SmC}_{\mathrm{A}} \mathrm{P}_{\mathrm{A}}$ phase upon cooling from the isotropic phase. The presence of a less ordered phase below a more ordered one upon cooling can be observed in liquid crystals due to a large number of competing interactions. The phase sequence of a tilted lamellar phase below the columnar $\mathrm{B}_{1}$ phase has been reported previously. ${ }^{5,28}$ Such a phenomenon was observed several times in different bent-core mesogens ${ }^{5}$ with ester linking groups in the bending arms as well as for a mesogen with a cinnamonic group ${ }^{28 a}$ inserted between the carboxyl group and the outer phenyl rings. A structural study of a $\mathrm{B}_{1}$-SmCP phase transition has been published by Folcia et $\mathrm{al}^{28 \mathrm{cc}}$ for a bent-core mesogen with a biphenyl central core. It seems that for all compounds the character and orientation of the linking groups play an important role. One can explain such an uncommon sequence with conformational changes in molecular arms being under way upon cooling, probably determined by the arrangement of the linkage groups. As was explained by Coleman et al. ${ }^{29}$ the $\mathrm{B}_{1}$ phase is driven by a polarization splay connected with a defect array. In the $\mathrm{B}_{1}$ phase, the layers are displaced at the defect boundaries.

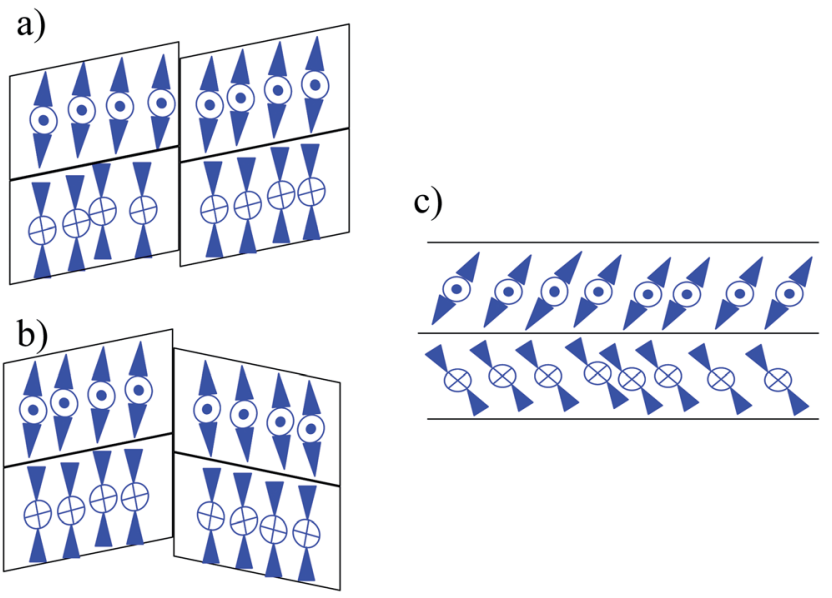

Fig. 9 Schematic organization of blocks (in the cross-section view) of a columnar $B_{1}$ phase: (a) and (b) are two possibilities, which cannot be distinguished between; (c) anticlinic and antiferroelectric organization of the tilted molecules in the neighbouring layers for the $\mathrm{SmC}_{A} \mathrm{P}_{\mathrm{A}}$ phase. 
Nevertheless, with the conventional non-resonant XRD we are not able to describe all the details about the molecular arrangement in the studied columnar $\mathrm{B}_{1}$ phase. $^{30} \mathrm{We}$ can propose a simple scheme to show how the molecules can be organized in a columnar phase (Fig. 9a and b show two alternative arrangements in the $\mathrm{B}_{1}$ phase, which cannot be recognized by X-ray analysis). Molecular ribbons can slide and at the phase transition the shift between them can disappear so the structure degenerates into a layered system (Fig. 9c). According to the switching behaviour and electro-optical properties the lamellar phase (confirmed by XRD results) can be unambiguously attributed to the $\mathrm{SmC}_{\mathrm{A}} \mathrm{P}_{\mathrm{A}}$ phase (Fig. 9c).

It is worth mentioning that a phase transition from the undulated/modulated ground structure to a switchable SmCP phase can be also induced by an electric field. ${ }^{31}$ We did not observe any textural modification under the applied electric field in the upper columnar phase (above $T=138{ }^{\circ} \mathrm{C}$ ). We observed a clear phase transition from the columnar to the lamellar structure upon cooling by XRD methods and the clear anomaly was also detected by DSC. We expect that in our case the layer undulation is more sensitive to the temperature change than to the applied electric field.

Photosensitivity due to the presence of an azo-unit was also found in the studied compounds. In the solution of the studied molecules the trans-cis isomerization takes place under illumination with UV-light. We have not studied light induced changes in the mesophases systematically. We are aware of the fact that the alignment of the molecules can be disturbed when we induce the trans-cis isomerization process by strong UV light in the mesophase. We intend to use the studied molecules as a photosensitive dopant and this issue will be studied elsewhere.

All lengthened molecules, $\mathbf{A} / \mathbf{2} / \mathbf{3}, \mathbf{A} / \mathbf{3} / \mathbf{1}, \mathbf{A} / \mathbf{3} / \mathbf{2}$ and $\mathbf{A} / \mathbf{3} / \mathbf{3}$, form the columnar $\mathrm{B}_{1}$ mesophase showing a rectangular cell with a similar dimension in the ribbon cross-section. The compounds differ only in the $b$ parameter (Table 2), which corresponds to the molecular length. For bent-shaped mesogens the lamellar phases are preferred when increasing the length of the terminal alkyl chain. ${ }^{11,28 d}$ In our case, the lengthening of the arms is caused by adding a phenyl ring that substantially changes the character of the molecular packing. More probably, the packing character is then more determined by a dipole moment and its orientation with respect to the whole molecular shape.

To summarize the results, we present how the overall molecular shape influences the type of self-organization. Hockey-stick molecules with strong asymmetry between the arms exhibit only calamitic mesophases. Lengthening the molecular arms leads to the restriction of the molecular rotation around the long axis. It results in the close packing of the molecules and thus mesophases typical for bent-core systems can be observed. We can confirm that the mesoscale organization strictly depends upon the molecular shape.

\section{Acknowledgements}

This work was supported by the Czech Science Foundation (projects P204/11/0723 and 13-14133S) and the Academy of Sciences of the Czech Republic (project M100101211).

\section{References}

1 T. Niori, T. Sekine, J. Watanabe, T. Furukawa and H. Takezoe, J. Mater. Chem., 1996, 6, 1231-1234.

2 R. Amaranatha Reddy and C. Tschierske, J. Mater. Chem., 2006, 16, 907-961.

$3 \mathrm{H}$. Takezoe and Y. Takanishi, Japanese Journal of Applied Chemistry, 2006, 45, 597-625.

4 W. Weissflog, H. N. Shreenivasa Murthy, S. Diele and G. Pelzl, Philos. Trans. R. Soc., A, 2006, 364, 2657-2679.

5 W. Weissflog, G. Naumann, B. Košata, M. W. Schröder, A. Eremin, S. Diele, Z. Vakhovskaya, H. Kresse, R. Friedemann, S. Ananda Rama Krishnan and G. Pelzl, J. Mater. Chem., 2005, 15, 4328-4337.

6 C. H. Keith, A. Lehmann, U. Baumeister, M. Prehm and C. Tschierske, Soft Matter, 2010, 6, 1704-1721.

7 L. A. Madsen, T. J. Dingemans, M. Nakata and E. T. Samulski, Phys. Rev. Lett., 2004, 92, 145505.

8 M. Kohout, J. Svoboda, V. Novotná, D. Pociecha, M. Glogarová and E. Gorecka, J. Mater. Chem., 2009, 19, 3153-3160.

9 M. Kohout, J. Svoboda, V. Novotná, M. Glogarová and D. Pociecha, Liq. Cryst., 2010, 37, 987-996.

10 M. Kohout, J. Svoboda, V. Novotná and D. Pociecha, Liq. Cryst., 2011, 38, 1099-1110.

11 G. Pelzl, S. Diele and W. Weissflog, Adv. Mater., 1999, 11, 707-724.

12 K. C. Majumdar, P. K. Shyam and S. Chakravorty, Liq. Cryst., 2010, 37, 1237-1243.

13 V. Prasad, S.-W. Kang and S. Kumar, J. Mater. Chem., 2003, 13, 1259-1264.

14 V. Prasad and A. Jákli, Liq. Cryst., 2004, 31, 473-479.

15 K. Gomola, L. Guo, D. Pociecha, F. Araoke, K. Ishikawa and H. Takezoe, J. Mater. Chem., 2010, 20, 7944-7952.

16 V. Kozmík, M. Horčic, J. Svoboda, V. Novotná and D. Pociecha, Liq. Cryst., 2012, 39, 943-955.

17 C. L. Folcia, I. Alonso, J. Ortega, J. Etxebarria, I. Pintre and M. Blanca Ros, Chem. Mater., 2006, 18, 4617-4626.

18 I. C. Pintre, N. Gimeno, J. L. Serrano, M. Blanca Ros, I. Alonso, C. L. Folcia, J. Ortega and J. Etxebarria, J. Mater. Chem., 2007, 17, 2219-2227.

19 N. G. Nagaveni, A. Roy and V. Prasad, J. Mater. Chem., 2012, 22, 8948-8959.

20 (a) M. Vijaysrinivasan, P. Kannan and A. Roy, Liq. Cryst., 2012, 39, 1465-1475; (b) N. G. Nagaveni, V. Prasad and A. Roy, Liq. Cryst., 2013, 40, 1001-1015.

21 N. G. Nagaveni, P. Raghuvanshi, A. Roy and V. Prasad, Liq. Cryst., 2013, 40, 1001-1005.

22 (a) R. Stannarius, J. Li and W. Weissflog, Phys. Rev. E: Stat., Nonlinear, Soft Matter Phys., 2003, 90, 025502; (b) B. Das, S. Grande, W. Weissflog, A. Eremin, M. W. Schröder, G. Pelzl, S. Diele and H. Kresse, Liq. Cryst., 2003, 30, 529539.

23 A. Chakraborty, B. Das, S. Findeisen-Tandel, M.-G. Tamba, U. Baumeister, H. Kresse and W. Weissflog, Liq. Cryst., 2011, 38, 1085-1097.

24 F. C. Yu and L. J. Yu, Chem. Mater., 2006, 18, 5410-5420. 
Paper

View Article Online

25 V. Novotná, J. Žurek, V. Kozmík, J. Svoboda and M. Glogarová, Liq. Cryst., 2008, 35, 1023-1036.

26 (a) S. Radhika, H. T. Srinivasa and B. K. Sadashiva, Liq. Cryst., 2011, 38, 785-792; (b) P. Sathyanarayana, S. Radhika, B. K. Sadashiva and S. Dhara, Soft Matter, 2012, 8, 2322-2327.

27 M. Glogarova, F. Hampl, L. Lejcek, V. Novotna, J. Svoboda and M. Cigl, J. Chem. Phys., 2010, 133, 221102.

28 (a) K. Gomola, L. Guo, S. Dhara, Y. Shimbo, E. Gorecka, D. Pociecha, J. Mieczkowski and H. Takezoe, J. Mater. Chem., 2009, 19, 4240-4247; (b) G. Pelzl, M. G. Tamba, S. Findeisen-Tandel, M. W. Schroeder, U. Baumeister, S. Diele and W. Weissflog, J. Mater. Chem., 2008, 18, 30173031; (c) C. L. Folcia, J. Etxebaria, J. Ortega and M. B. Ros, Phys. Rev. E: Stat., Nonlinear, Soft Matter Phys., 2006, 74, 031702; (d) H. N. Shreenivasa Murthy and B. K. Sadashiva, Liq. Cryst., 2004, 31, 1347.
29 D. A. Coleman, C. D. Jones, M. Nakata, N. A. Clark, D. M. Walba, W. Weissflog, K. Fodor-Csorba, J. Watanabe, V. Novotna and V. Hamplova, Phys. Rev. E: Stat., Nonlinear, Soft Matter Phys., 2008, 77, 021703.

30 C. L. Folcia, J. Ortega, J. Etxebarria, L. Dong Pan, S. Wang, C. C. Huang, V. Ponsinet, P. Barois, R. Pindak and N. Gimeno, Phys. Rev. E: Stat., Nonlinear, Soft Matter Phys., 2011, 84, 010701.

31 (a) R. Amaranatha Reddy, U. Baumeister, J. Lorenzo Chao, H. Kresse and C. Tschierske, Soft Matter, 2010, 6, 3883-3897; (b) J. Ortega, C. L. Folcia, J. Etxebarria, J. Martinez-Perdiguero, J. A. Gallastegui, P. Ferrer, N. Gimeno and M. Blanca Ros, Phys. Rev. E: Stat., Nonlinear, Soft Matter Phys., 2011, 84, 021707; (c) J. Kirchhoff and L. S. Hirst, Appl. Phys. Lett., 2007, 90, 161905.

This journal is @ The Royal Society of Chemistry 2013

J. Mater. Chem. C, 2013, 1, 7560-7567 | 7567 